\title{
Clinical study of the time of repeated computed tomography and replanning for patients with nasopharyngeal carcinoma
}

\author{
Xiujuan Gai ${ }^{1,2, *}$, Yumei Wei ${ }^{2, *}$, Hengmin $\mathrm{Tao}^{3,4}$, Jian Zhu ${ }^{5}$ and Baosheng $\mathrm{Li}^{2}$ \\ ${ }^{1}$ School of Medicine and Life Sciences, University of Jinan-Shandong Academy of Medical Sciences, Shandong, China \\ ${ }^{2}$ Department of Radiation Oncology VI, Shandong Cancer Hospital Affiliated to Shandong University, Jinon, Shandong, China \\ ${ }^{3}$ Shandong Provincial Hospital affiliated to Shandong University, Shandong, China \\ ${ }^{4}$ Shandong Provincial Western Hospital, Shandong, China \\ ${ }^{5}$ Department of Radiation Oncology, Shandong Cancer Hospital Affiliated to Shandong University, Shandong, China \\ * These authors have contributed equally to this work \\ Correspondence to: Baosheng Li, email: baoshli1963@163.com
}

Keywords: nasopharyngeal carcinoma (NPC), cone beam computed tomography (CBCT), gross tumor volume (GTV), parotid gland, displacement

Received: November 14, $2016 \quad$ Accepted: March 09, $2017 \quad$ Published: March 31, 2017

Copyright: Gai et al. This is an open-access article distributed under the terms of the Creative Commons Attribution License (CC-BY), which permits unrestricted use, distribution, and reproduction in any medium, provided the original author and source are credited.

\section{ABSTRACT}

Purpose: To study the necessity of repeat computed tomography (CT) scan and replanning and know a more accurate time using weekly kilovoltage cone beam computed tomography (kV-CBCT) scans for patients with nasopharyngeal carcinoma (NPC) during radiotherapy.

Methods and Materials: Thirteen NPC patients treated with IMRT were enrolled into this prospective study. Weekly pretreatment kV-CBCT scans were performed on the $1^{\text {st }}, 6^{\text {th }}, 11^{\text {st }}, 16^{\text {th }}, 21^{\text {st }}$ and $26^{\text {th }}$ radiation time, respectively. Target delineations were contoured on all fractionated CBCT images, including the gross tumor volume of the primary nasopharyngeal tumor (GTVnx) and parotid glands. The volumes of GTVnx and parotid glands were calculated automatically using the Pinnacle ${ }^{3} 8.0$ system. Compared to the original GTVnx, the percentage of shrinking volume $(\Delta P) \geq 50 \%$ was considered significantly.

Results: As the radiation proceeding, the GTVnx had a trend of shrinkage. Of all 13 patients, 11 cases $(84.6 \%)$ had the volume shrinking $\geq 50 \%$ before the $21^{\text {st }}$ radiation and 12 cases $(92.3 \%)$ before the $26^{\text {th }}$ radiation. And the parotid volume decreased significantly in the first four-week radiation, $6.45 \pm 3.16 \mathrm{~cm}^{3}$ (range, 3.06$\left.13.9 \mathrm{~cm}^{3}\right)$ for the left parotid gland and $5.78 \pm 2.39 \mathrm{~cm}^{3}\left(\right.$ range, $\left.2.70-11.2 \mathrm{~cm}^{3}\right)$ for the right. Furthermore, only a little displacement occurred to bilateral parotid glands.

Conclusion: The replanning for NPC patients with IMRT is necessary, and the time between the $21^{\text {st }}$ to $25^{\text {th }}$ radiations is appropriate.

\section{INTRODUCTION}

Nasopharyngeal carcinoma (NPC) is commonly seen in endemic populations from some regions of the world, especially, the Southern China. Due to the anatomically challenging location and its high radiosensitivity, radiation therapy (RT) has been recognized as the main curative treatment modality for locoregionally confined NPC [1]. Over the past decade, intensity-modulated radiation therapy (IMRT) has acquired excellent popularity in the treatment of NPC, because of its high efficacy of local control and decreased treatment toxicity. Compared with conventional radiotherapy, IMRT offers improved dose conformity to tumor target coverage with a relative sparing of sensitive normal tissues [2-5]. With the sharp dose drop-offs common to IMRT plans, consistent and accurate dose distribution to tumor targets and their surrounding critical organs are crucial $[6,7]$. However, in the course of treatment, most of NPC patients undergoing IMRT have significant changes in anatomical structures, including tumor shrinkage and/or surrounding critical organs shrinkage. And these led to decreased doses to tumor targets and increased to critical structures $[8,9]$. For example, the parotid glands undertake the major 
role in salivary secretion. And xerostomia is one of the most common side effects caused by amount of radiation delivered to parotid glands, which would affect patients' quality of life. So it is of great significance to grasp changes in volume and displacement of tumor targets and the parotid glands throughout the IMRT treatment course.

With the development of radiotherapy technology, kilovoltage $(\mathrm{kV})$ cone beam computed tomography (CBCT) imaging system installed on a medical linear accelerator has been introduced. It has become an important technique for realizing image-guided radiation therapy. During the course of fractionated radiotherapy, the $\mathrm{kV}-\mathrm{CBCT}$ could not only assess patient setup errors contrasted with the bony anatomy and soft-tissues, but monitor volume and displacement changes of tumor targets and relative critical organs. A recent study has reported that for NPC patients the second CT scan and replanning before the $25^{\text {th }}$ fraction of IMRT are benefit to ensure appropriate doses to tumor target volumes and surrounding normal tissues [10].

In order to study further the necessity of repeat CT scan and replanning and know the more accurate time for patients with NPC, we aimed at investigating tumor targets' volume and parotid glands' volume and displacement changes using weekly $\mathrm{kV}$-CBCT scans during the course of IMRT.

\section{RESULTS}

The volumes of GTVnx on fractionated CBCT images were all shown in Table 2, while Table 3 and Table 4 showed all values of $\Delta \mathrm{GTV}$ and $\Delta \mathrm{P}$, respectively. As the radiation proceeding, the GTVnx had a trend of shrinkage. In the first four-week radiotherapy, the total shrinking volumes $\left(\Delta \mathrm{GTV}_{21}\right)$ ranged from $7.2 \mathrm{~cm}^{3}$ to 20.7 $\mathrm{cm}^{3}$ (mean, $\left.13.7 \mathrm{~cm}^{3}\right)$, with the percentage $\left(\Delta \mathrm{P}_{21}\right)$ from $19.3 \%$ to $69.1 \%$ (median, $54.8 \%$ ). And before the $26^{\text {th }}$ radiation, the shrinking volumes $\left(\Delta \mathrm{GTV}_{26}\right)$ ranged from $7.6 \mathrm{~cm}^{3}$ to $27.1 \mathrm{~cm}^{3}$ (mean, $19.6 \mathrm{~cm}^{3}$ ), with the percentage $\left(\Delta \mathrm{P}_{26}\right)$ from $20.4 \%$ to $85.3 \%$ (median, $82.8 \%$ ). Meantime, the correlation analysis showed that the percentage of shrinkage was related to the initial volume of GTVnx (r $=-0.623, P=0.023)$, but the absolute decline in values were not connected with it. In addition, of all 13 patients, only one person $(1 / 13)$ had the volume shrinking $\geq 50 \%$ $(52 \%)$ before the $16^{\text {th }}$ radiation, but 11 cases $(84.6 \%)$ before the $21^{\text {st }}$ radiation and 12 cases $(92.3 \%)$ before the $26^{\text {th }}$ radiation, including 11 cases $(84.6 \%)$ decreased $\geq$ $70 \%$ and 9 cases $(69.2 \%)$ decreased $\geq 80 \%$. Furthermore, Figure 2 and Figure 3 displayed the tendency of mean $\triangle \mathrm{GTV}$ and $\Delta \mathrm{P}$ as the radiation, both with a higher rate between the $21^{\text {st }}$ radiation and the $26^{\text {th }}$ radiation.

On the other hand, the parotid volume decreased significantly during radiotherapy, as displayed in Figure 4, and with all values showed in Table 5 and Table 6 . In the first four-week radiotherapy, the total shrinked volumes $\left(\Delta \mathrm{V}_{21}\right)$ of the left and right parotid glands were $6.45 \pm$ $3.16 \mathrm{~cm}^{3}$ (range, $3.06-13.9 \mathrm{~cm}^{3}$ ) and $5.78 \pm 2.39 \mathrm{~cm}^{3}$ (range, $\left.2.70-11.2 \mathrm{~cm}^{3}\right)$, respectively. And before the $26^{\text {th }}$ radiation, the volume reduction $\left(\Delta \mathrm{V}_{26}\right)$ was $7.20 \pm 3.33 \mathrm{~cm}^{3}$ (range, $3.75-14.1 \mathrm{~cm}^{3}$ ) and $6.34 \pm 2.50 \mathrm{~cm}^{3}$ (range, $2.88-12.0 \mathrm{~cm}^{3}$ ) for the left and right parotid glands, respectively. When measured as a percentage of the initial volume before the $21^{\text {st }}$ radiation, the average volume reduction was $41.7 \%$ and $31.6 \%$ for the left and right parotid glands, respectively, and before the $26^{\text {th }}$ radiation, the percentage
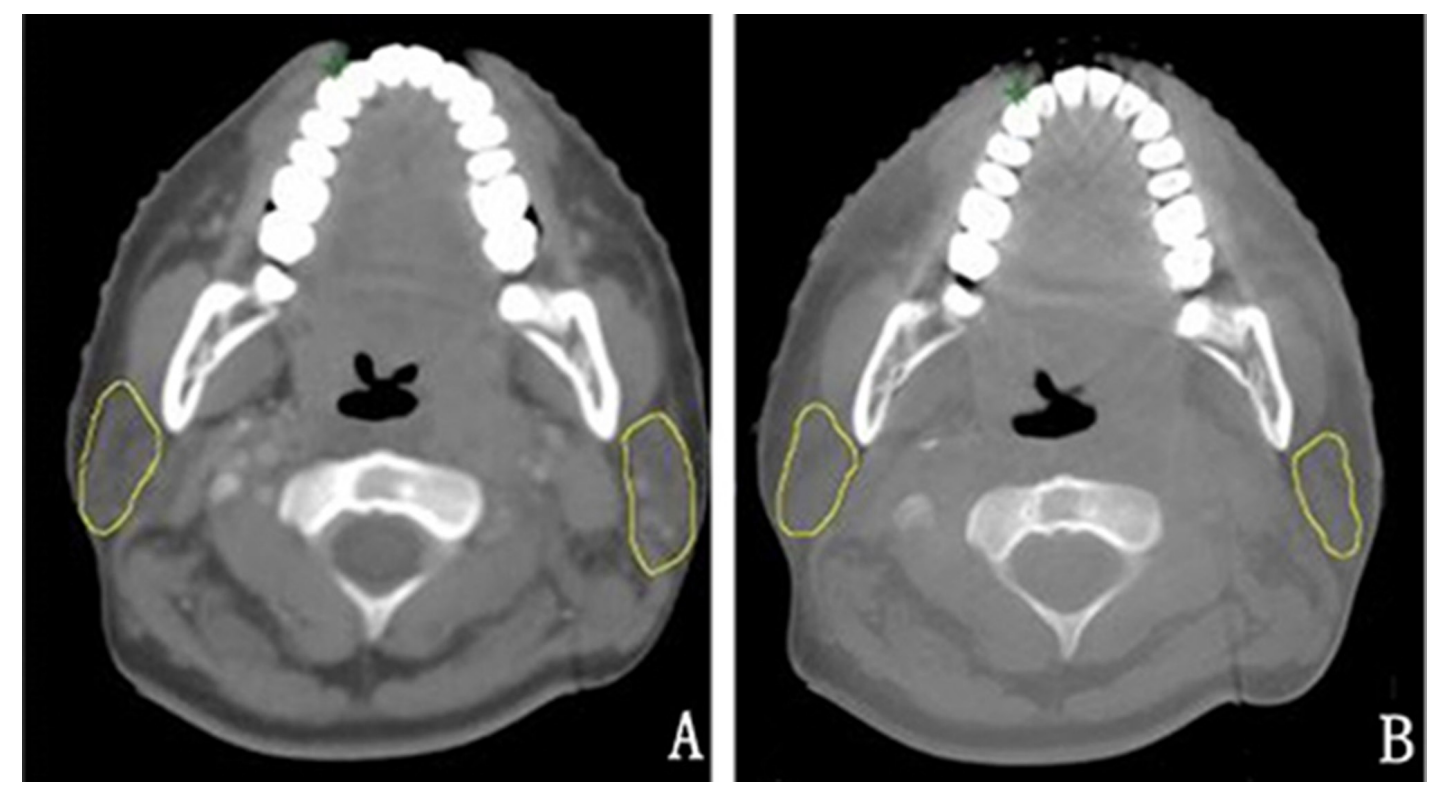

Figure 1: The contrast of the nasopharynx in the transverse section between the planning CT image and CBCT image before the first radiation from one patient. A. the planning CT B. CBCT before the first radiation. 
Table 1: Patient characteristics and tumor stage

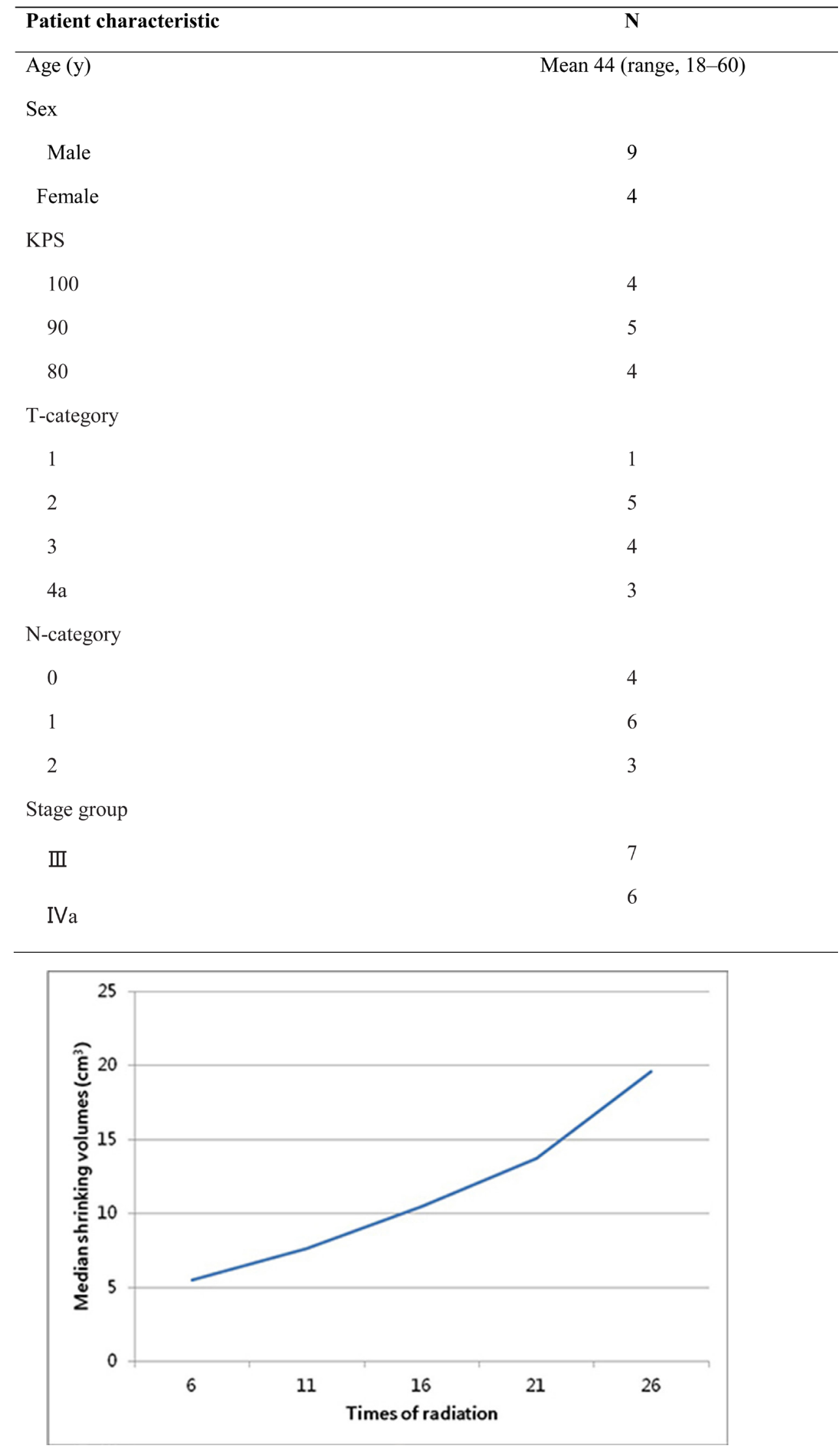

Figure 2: The tendency of median volumetric changes of GTVnx between the fractionated volume and original volume. 
Table 2: The volumes of GTVnx of all NPC patients on fractionated CBCT images

\begin{tabular}{|c|c|c|c|c|c|c|}
\hline No. $v\left(\mathrm{~cm}^{3}\right)$ & GTV1 & GTV6 & GTV11 & GTV16 & GTV21 & GTV26 \\
\hline 1 & 37.8 & 30.3 & 27.5 & 20.3 & 17.1 & 10.9 \\
\hline 2 & 21.5 & 16.9 & 14.4 & 13.1 & 10.1 & 3.7 \\
\hline 3 & 20.4 & 15.5 & 15.1 & 9.8 & 6.3 & 3.2 \\
\hline 4 & 16.3 & 12.1 & 10.6 & 8.9 & 7.2 & 3.8 \\
\hline 5 & 30.4 & 23.4 & 21.6 & 17.8 & 12.8 & 4.5 \\
\hline 6 & 22.8 & 16.8 & 15.4 & 12.7 & 10.3 & 3.5 \\
\hline 7 & 24.3 & 18.3 & 16.5 & 12.9 & 11.2 & 4.1 \\
\hline 8 & 27.2 & 21.0 & 17.1 & 14.3 & 11.6 & 4.0 \\
\hline 9 & 18.8 & 14.1 & 12.7 & 11.7 & 8.6 & 3.4 \\
\hline 10 & 25.8 & 18.4 & 16.5 & 13.6 & 11.4 & 4.8 \\
\hline 11 & 32.4 & 25.4 & 21.6 & 19.8 & 14.2 & 5.3 \\
\hline 12 & 37.3 & 37.1 & 32.9 & 32.2 & 30.1 & 29.7 \\
\hline 13 & 41.7 & 35.7 & 35.4 & 33.6 & 27.5 & 20.4 \\
\hline
\end{tabular}

(GTVnx: Gross tumor volume of primary nasopharyngeal tumor, NPC: Nasopharyngeal carcinoma, CBCT: Cone beam computed tomography, GTV: Gross tumor volume)

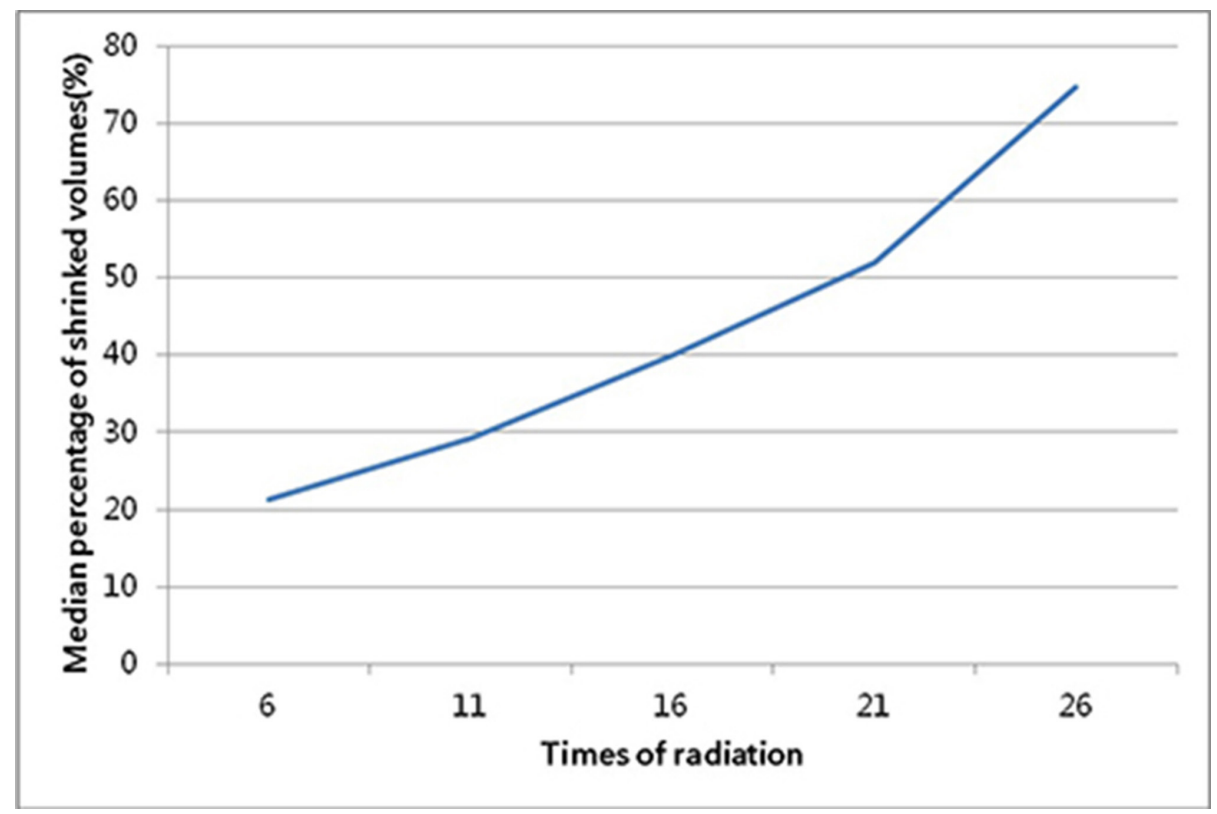

Figure 3: The variation tendency of mean percentage of shrinking volume for the fractionated volume compared to the original volume. 
Table 3: Volumetric changes of GTVnx beween each fractionated volume and the original volume

\begin{tabular}{|c|c|c|c|c|c|}
\hline No. $\Delta v(\mathrm{~cm} 3)$ & $\Delta$ GTV6 & $\Delta$ GTV11 & $\Delta$ GTV16 & $\Delta G T V 21$ & $\Delta$ GTV26 \\
\hline 1 & 7.5 & 10.3 & 17.5 & 20.7 & 26.9 \\
\hline 2 & 4.6 & 7.1 & 8.4 & 11.4 & 17.8 \\
\hline 3 & 4.9 & 5.3 & 10.6 & 14.1 & 17.2 \\
\hline 4 & 4.2 & 5.7 & 7.4 & 9.1 & 12.5 \\
\hline 5 & 7.0 & 8.8 & 12.6 & 17.6 & 25.9 \\
\hline 6 & 6.0 & 7.4 & 10.1 & 12.5 & 19.3 \\
\hline 7 & 6.0 & 7.8 & 11.4 & 13.1 & 20.2 \\
\hline 8 & 6.2 & 10.1 & 12.9 & 15.6 & 23.2 \\
\hline 9 & 4.7 & 6.1 & 7.1 & 10.2 & 15.4 \\
\hline 10 & 7.4 & 9.3 & 12.2 & 14.4 & 21.0 \\
\hline 11 & 7.0 & 10.8 & 12.6 & 18.2 & 27.1 \\
\hline 12 & 0.2 & 4.4 & 5.1 & 7.2 & 7.6 \\
\hline 13 & 6.0 & 6.3 & 8.1 & 14.2 & 21.3 \\
\hline
\end{tabular}

(GTVnx: Gross tumor volume of primary nasopharyngeal tumor, $\Delta \mathrm{GTV}$ : Volumetric changes of GTVnx)

Table 4: Percentage of shrinking volume for the fractionated volume compared to the original volume

\begin{tabular}{|c|c|c|c|c|c|}
\hline No. $P(\%)$ & $\Delta \mathrm{P}_{6}$ & $\Delta \mathrm{P}_{11}$ & $\Delta \mathrm{P}_{16}$ & $\Delta \mathrm{P}_{21}$ & $\Delta \mathrm{P}_{26}$ \\
\hline 1 & 19.8 & 27.2 & 46.3 & 54.8 & 71.2 \\
\hline 2 & 21.4 & 33.0 & 39.1 & 53.0 & 82.8 \\
\hline 3 & 24.0 & 26.0 & 52.0 & 69.1 & 84.3 \\
\hline 4 & 25.8 & 35.0 & 45.4 & 55.8 & 76.7 \\
\hline 5 & 23.0 & 28.9 & 41.4 & 57.9 & 85.2 \\
\hline 6 & 26.3 & 32.5 & 44.3 & 54.8 & 84.6 \\
\hline 7 & 24.7 & 32.1 & 46.9 & 53.9 & 83.1 \\
\hline 8 & 22.8 & 37.1 & 47.4 & 57.4 & 85.3 \\
\hline 9 & 25.0 & 32.4 & 37.8 & 54.3 & 81.9 \\
\hline 10 & 28.7 & 36.0 & 47.3 & 55.8 & 81.4 \\
\hline 11 & 21.6 & 33.3 & 38.9 & 56.2 & 83.6 \\
\hline 12 & 0.5 & 11.8 & 13.7 & 19.3 & 20.4 \\
\hline 13 & 14.4 & 15.1 & 19.4 & 34.1 & 51.1 \\
\hline
\end{tabular}

$(\Delta \mathrm{P}$ : Percentage of shrinking volume) 
Table 5: Volumes of the left parotid gland of all NPC patients on fractionated CBCT images

\begin{tabular}{ccccccc}
\hline $\begin{array}{c}\text { V(cm } \\
\text { No. }\end{array}$ & $\mathbf{1}$ & $\mathbf{6}$ & $\mathbf{1 1}$ & $\mathbf{1 6}$ & $\mathbf{2 1}$ & $\mathbf{2 6}$ \\
\hline 1 & 16.28 & 14.75 & 12.91 & 12.04 & 11.87 & 11.20 \\
2 & 16.86 & 16.28 & 15.8 & 14.54 & 13.8 & 13.11 \\
3 & 26.33 & 25.22 & 23.49 & 23.03 & 22.67 & 21.9 \\
4 & 25.66 & 24.66 & 21.99 & 21.87 & 21.17 & 21.04 \\
5 & 11.23 & 10.04 & 8.94 & 6.46 & 6.12 & 5.34 \\
6 & 16.04 & 6.49 & 4.99 & 2.47 & 2.10 & 1.98 \\
7 & 9.54 & 7.89 & 7.80 & 4.05 & 3.78 & 3.18 \\
8 & 13.42 & 8.86 & 5.18 & 3.15 & 2.98 & 2.65 \\
9 & 22.8 & 16.78 & 13.42 & 13.26 & 12.52 & 9.67 \\
10 & 16.79 & 15.78 & 14.65 & 13.21 & 10.57 & 9.34 \\
11 & 15.43 & 13.23 & 12.32 & 10.45 & 9.87 & 9.12 \\
12 & 13.56 & 11.76 & 11.21 & 10.2 & 8.65 & 8.12 \\
13 & 14.78 & 13.56 & 11.89 & 9.98 & 8.78 & 8.45
\end{tabular}

(NPC: Nasopharyngeal carcinoma, CBCT: Cone beam computed tomography)

Table 6: Volumes of the right parotid gland of all NPC patients on fractionated CBCT images

\begin{tabular}{|c|c|c|c|c|c|c|}
\hline No. $V\left(\mathrm{~cm}^{3}\right)$ & 1 & 6 & 11 & 16 & 21 & 26 \\
\hline 1 & 17.97 & 16.9 & 14.47 & 13.56 & 12.53 & 11.89 \\
\hline 2 & 19.98 & 17.44 & 15.89 & 15.39 & 14.7 & 14.1 \\
\hline 3 & 34.12 & 32.81 & 29.63 & 29.48 & 27.6 & 26.8 \\
\hline 4 & 24.48 & 24.42 & 22.06 & 21.9 & 21.78 & 21.6 \\
\hline 5 & 12.38 & 10.85 & 10.37 & 9.98 & 8.78 & 8.56 \\
\hline 6 & 17.92 & 17.52 & 13.01 & 10.13 & 9.33 & 9.04 \\
\hline 7 & 11.59 & 11.28 & 10.32 & 9.34 & 8.76 & 8.10 \\
\hline 8 & 15.88 & 9.51 & 6.82 & 5.93 & 4.68 & 3.84 \\
\hline 9 & 20.34 & 16.51 & 14.57 & 13.84 & 13.52 & 13.42 \\
\hline 10 & 18.9 & 16.78 & 15.46 & 13.78 & 11.29 & 10.34 \\
\hline 11 & 25.34 & 23.98 & 21.76 & 20.67 & 19.98 & 19.21 \\
\hline 12 & 12.67 & 11.89 & 10.45 & 8.78 & 8.21 & 7.89 \\
\hline 13 & 21.67 & 20.56 & 19.67 & 17.21 & 16.89 & 16.01 \\
\hline
\end{tabular}

(NPC: Nasopharyngeal carcinoma, CBCT: Cone beam computed tomography) 
was $46.2 \%$ and $34.6 \%$, respectively. Although the decrease in the volume of left parotid is larger than that of right parotid, there were no statistical significance, with $P$ $=0.55$ and $P=0.46$ after the first four-week and the $5^{\text {th }}$ week, respectively. No correlation was found between the original volumes and shrinking volumes. But there was obviously positive correlation between the absolute and relative shrinkage of parotid volumes and the planned mean dose $\left(\mathrm{D}_{\text {mean }}\right)$ for bilateral parotids, with the value of $r$ and $P$ displayed in Table 7. Furthermore, Figure 5 showed the volumetric variation trend of bilateral parotid glands, respectively, with volumes decreasing significantly in the first four-week radiation for bilateral parotid glands.

Figure 6 displayed the variation trend of average $\mathrm{D}_{\text {mean }}$ delivered to bilateral parotid glands per fraction, respectively. It showed the tendency of rising after the $11^{\text {st }}$ radiation. And the percentages of increased average $\mathrm{D}_{\text {mean }}$ of the left parotid gland were $14.6 \%$ and $45.3 \%$ at the $21^{\text {st }}$ and $26^{\text {th }}$ radiation, respectively, with $9.4 \%$ and $11.4 \%$ for the right. Meanwhile, median displacement of the parotid glands were displayed in Table 8 . Only a little displacement occurred to bilateral parotid glands. But a trend to shift medially for both the left and right parotid gland could also been demonstrated.
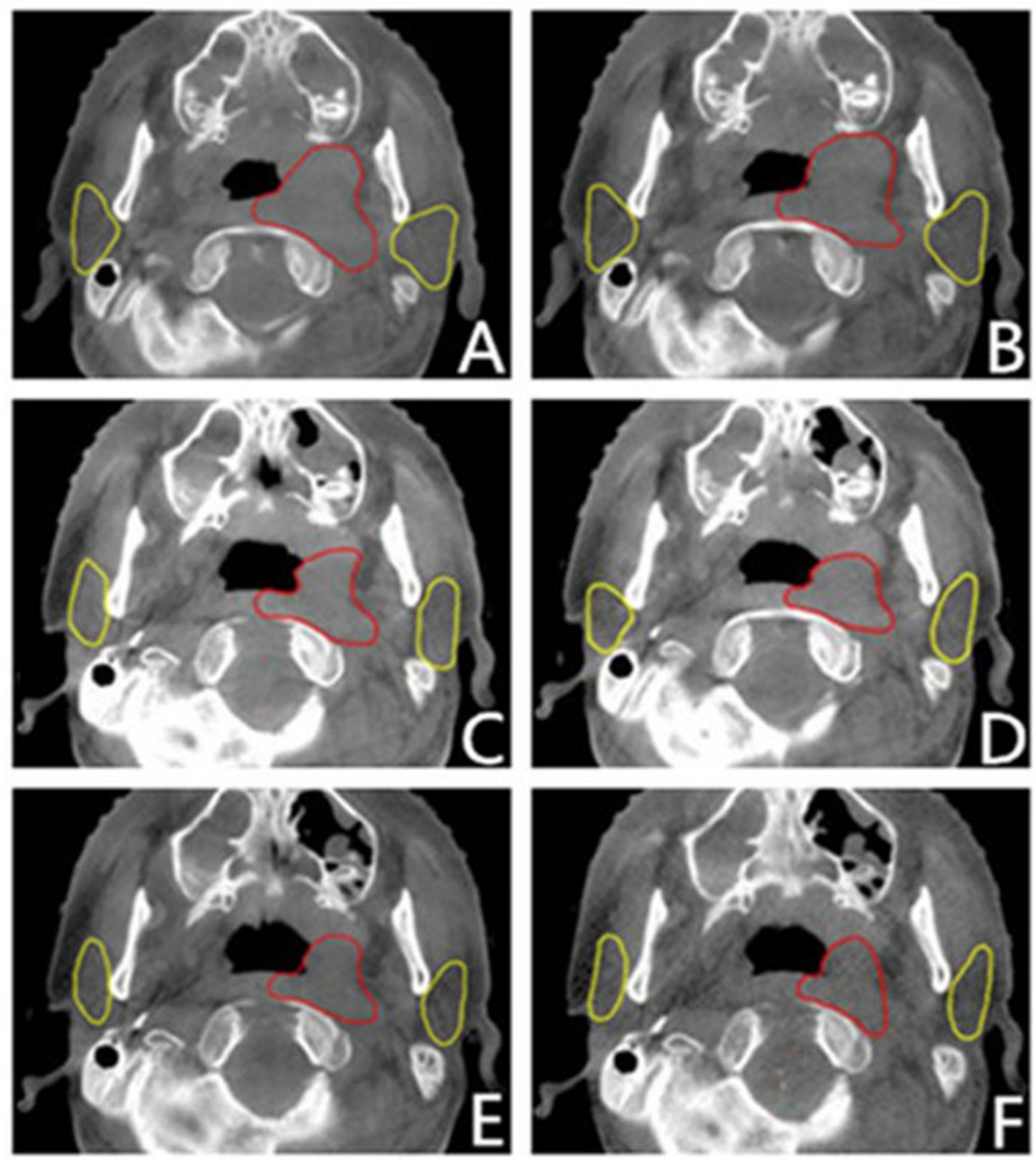

Figure 4: CBCT images performed before the $1^{\text {st }}, 6^{\text {th }}, 11^{\text {st }}, 16^{\text {th }}, 21^{\text {st }}$ and $26^{\text {th }}$ radiation time from one patient, corresponding to letter A, B, C, D, E, F. (In this level, the GTV and parotid glands were showed typically and clearly. The red outlines were GTV, and the yellow were parotids.) 
Table 7: The correlation between the shrinkage of parotid volume and the planned mean dose for bilateral parotids

\begin{tabular}{lllllr}
\hline Shrinkage & Times of radiation & \multicolumn{2}{l}{ The left parotid } & \multicolumn{2}{l}{ The right parotid } \\
\cline { 3 - 6 } & & $\mathbf{r}$ & $\boldsymbol{P}$ & $\mathbf{r}$ & $\boldsymbol{P}$ \\
\hline Absolute & $21^{\text {th }}$ & 0.934 & $0.001^{*}$ & 0.916 & $0.001^{*}$ \\
& $26^{\text {th }}$ & 0.842 & $0.009^{*}$ & 0.925 & $0.001^{*}$ \\
\hline Relative & $21^{\text {th }}$ & 0.812 & 0.014 & 0.845 & $0.008^{*}$ \\
& $26^{\text {th }}$ & 0.769 & 0.018 & 0.850 & $0.008^{*}$
\end{tabular}

(Absolute: the shrinking volumes of parotids, Relative: the percentage of shrinking volumes compared with the original volumes, r: Pearson correlation coefficient, "*": Two-sided P-values of $<0.01$ were considered statistically significant.)

Table 8: Median distances between the parotid COM and the atlas COM

\begin{tabular}{lll}
\hline Times of treatment & \multicolumn{2}{c}{ Distances between the parotid COM and the atlas COM (cm) } \\
\cline { 2 - 3 } & \multicolumn{2}{c}{ Left Parotid } \\
\hline 1 & 7.14 & 7.29 \\
6 & 7.12 & 7.24 \\
11 & 7.11 & 7.23 \\
16 & 7.03 & 7.21 \\
21 & 7.01 & 7.20 \\
26 & 6.93 & 7.16
\end{tabular}

(COM: Center of mass)

\section{DISCUSSION}

Over the past decade, IMRT has become the routine treatment for patients with NPC disease. But most NPC patients would experience anatomic changes during the course of IMRT, mainly including shrinkage of the primary tumor and displacement changes of parotid glands [8-10]. Due to the sharp dose gradient of IMRT technique, these changes could cause a potential dosimetric impact, including decreased doses to tumor targets and increased to normal structures. Therefore, repeat CT scan and replanning are necessary. A previous study has reported that repeat CT scan and replanning before the $25^{\text {th }}$ radiation would significantly increase the tumor target dose coverage and reduce the normal structures' doses [10]. However, all repeat CT scan and replanning in this study were performed only before the $25^{\text {th }}$ radiation, compared to another study with an average interval of $39 \pm 11$ days (average, $19 \pm 6$ fractions) between the first radiation and the repeat CT scan [11]. To monitor changes of the tumor and critical organs timely during the process of radiotherapy and know a more accurate time for repeat $\mathrm{CT}$ scan and replanning, we conducted a pilot study by using the $\mathrm{kV}-\mathrm{CBCT}$ weekly. And in our study, the shrinking volumes and percentage of GTVnx in the first four-week radiotherapy were similar to the result of another previous study, with the range from $5.1 \mathrm{~cm}^{3}$ to $21.4 \mathrm{~cm}^{3}$ (median, $9.85 \mathrm{~cm}^{3}$ ) and from $23.0 \%$ to $58.2 \%$ (median, $27.15 \%$ ), respectively [12]. We also demonstrated that a significant shrinkage ( $\geq 50 \%$ ) occurred after the $20^{\text {th }}$ radiation, and a substantial proportion of reduction then happened between the $21^{\text {st }}$ and $25^{\text {th }}$ radiation. As a result, replanning between the $21^{\text {st }}$ and $25^{\text {th }}$ fraction during the IMRT is recommended.

Not only could changes in organ anatomy and tumor size cause target underdose during IMRT, but also overdose to OARs could lead to additional complications, like xerostomia caused by high radiation dose of exposure to parotid glands. Although the incidence of xerostomia reduced with the use of IMRT technique, volumetric reduction and displacement change could also result in severe dry mouth symptoms, affecting the quality of life for patients with NPC disease $[13,14]$. In our study, the 
parotid volume decreased gradually during radiotherapy, with the average reduction of $41.7 \%$ and $31.6 \%$ for the left and right parotid glands in the first four-week and of $46.2 \%$ and $34.6 \%$ after the $5^{\text {th }}$ week. However, another recent study reported that the average volume reduction was $29.47 \%$ and $24.47 \%$ for the left and right parotid glands at the end of radiation therapy [15], which were smaller than our results. And previously published studies demonstrated that parotid volumes had decreased little during the first 3-4 weeks [16, 17] and stabilized after the $25^{\text {th }}$ radiation [14]. Maybe the use of helical tomotherapy and radiotherapy combined with different chemotherapy or anti-EGFR Mab could explain it.
We found a rising trend of the average $\mathrm{D}_{\text {mean }}$ for bilateral parotids per fraction, with increased percentages of $45.3 \%$ and $11.4 \%$ for the left and right parotid glands at the $26^{\text {th }}$ radiation, respectively. And a previous study reported a similar result, with the accumulative average $\mathrm{D}_{\text {mean }}$ increasing of $11.38 \%$ at the end of radiation therapy for all enrolled parotid glands with helical tomotherapy [18]. The sharp increase of the average $\mathrm{D}_{\text {mean }}$ per fraction for the left parotid gland could be caused by the location of the primary tumors, which were mostly $(8 / 13)$ located on the left side of the body. In the light of the previous study, replanning was regarded to be necessary if absolute parotid doses were more than $10 \%$ compared to the initial

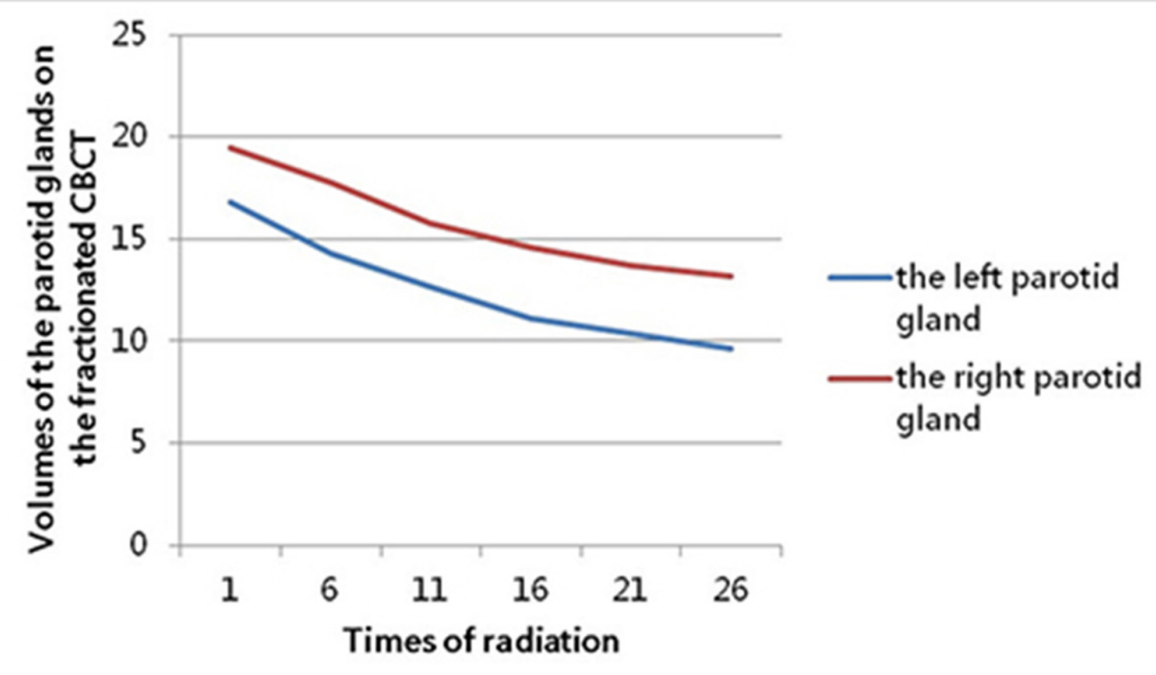

Figure 5: The variation tendency of the bilateral parotid glands' volume $\left(\mathrm{cm}^{3}\right)$ on the fractionated CBCT.

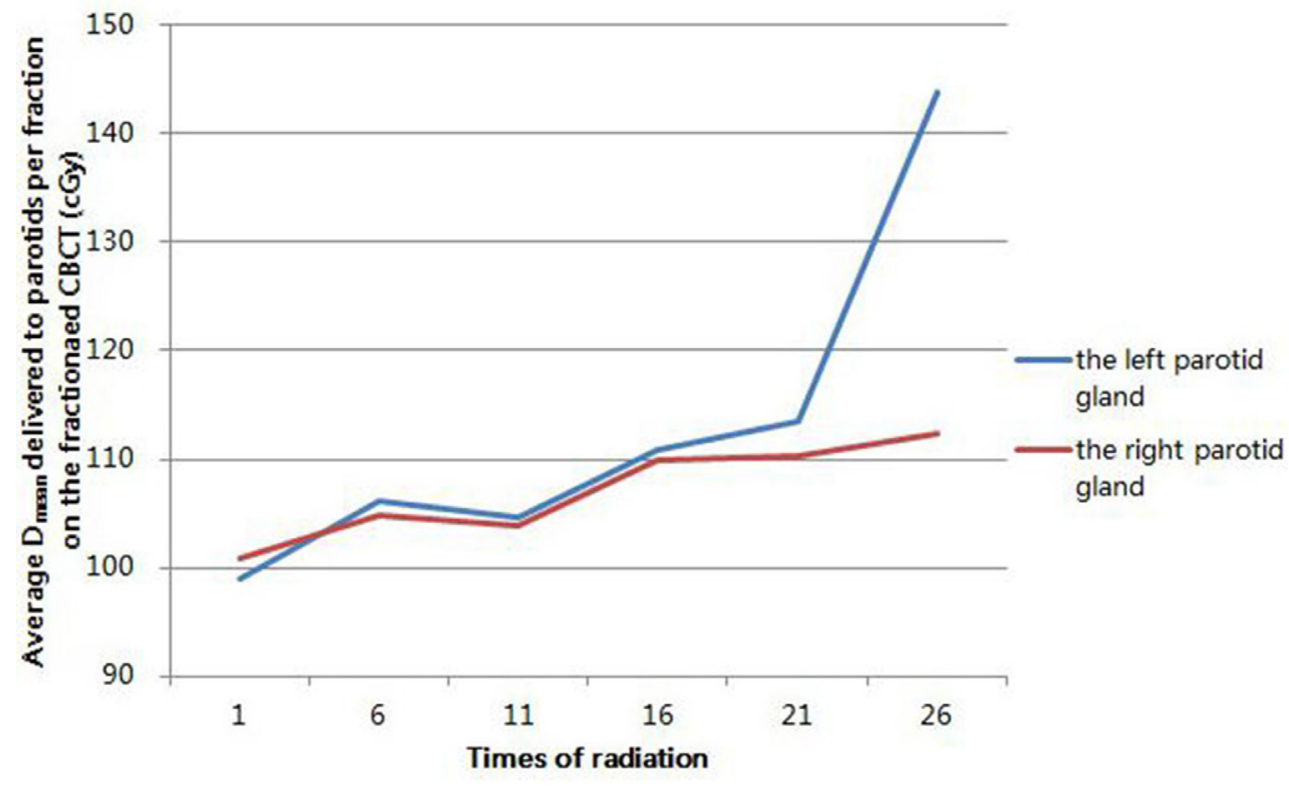

Figure 6: The variation trend of average mean doses $\left(\mathrm{D}_{\text {mean }}\right)$ delivered to bilateral parotid glands per fraction on the fractionated CBCT. 
planning dose. In our research, according to the shrinkage of GTVnx and the $\mathrm{D}_{\text {mean }}$ raise of parotids, it is necessary to execute replanning between the $21^{\text {st }}$ and $25^{\text {th }}$ fraction during the IMRT.

Similar to previous studies [19, 20], displacement changes in the parotid glands were not obvious in our study, which aimed at the displacement of parotid centers. Robar et al. [19] has reported that the parotid centers remained unchanged, though the outside boundaries shifted to the midline (with an average of $2.6 \mathrm{~mm}$ for the left parotid gland and $1.9 \mathrm{~mm}$ for the right parotid gland). And Vasquez Osorio et al [20] also found that displacement changes in the central region of parotid glands were minimal. We think that the changes in the peripheral region could be explained by the volumetric reduction of bilateral parotid glands. The traction caused by the shrinkage of tumors may result in the displacement of parotid centers. Most tumors (8/13) in our research were located on the left side of the body. And this could make the traction for the left parotid glands more than the one for the right, with the displacement of $0.21 \mathrm{~cm}$ for the left parotid and $0.13 \mathrm{~cm}$ for right after the $26^{\text {th }}$ radiation. But we think there was no significance for the little difference $(0.08 \mathrm{~cm})$.

\section{MATERIALS AND METHODS}

\section{Patient characteristics}

From January 2014 to April 2015, a total of 13 newly diagnosed patients at the Shandong Cancer Hospital Affiliated to Shandong University were enrolled into this prospective study. Eligible patients were individuals with histologically proven and locoregionally advanced (stage III-IVa) NPC. The disease was staged according to the 7th American Joint Commission on Cancer staging system. Patients specific characteristics are summarized in Table 1. CBCT is a generally accepted technique to monitor variations and decrease setup errors for NPC patients undergoing IMRT, so the authors advise that the Institutional Review Board deemed the study violated no ethics and the ethics approval was not imperative. Informed consent was provided by all patients before participation.

\section{Radiotherapy simulation}

Patients were immobilized with a thermoplastic head-and-shoulder mask in the supine position. Imagings were scanned with a slice thicknesses of $3 \mathrm{~mm}$ on a computed tomography simulator. Each scan was performed from the vertex to below the clavicles. The CT datasets were transmitted to the Pinnacle ${ }^{3} 8.0$ workstation through DICOM network for contouring targets and organs at risk (OARs).

\section{Target delineation and treatment planning}

The target delineation was contoured on the simulation CT images by one oncologist and confirmed by another oncologist. The gross tumor volume (GTV) included the primary nasopharyngeal tumor (GTVnx) and involved lymph nodes (GTVnd) as shown in the enhanced CT images, magnetic resonance imaging images and/or positron emission tomography. The clinical target volume 1 (CTV1) was defined as high-risk regions surrounding the primary tumor (including the entire nasopharynx, skull base, parapharyngeal space, posterior third of the nasal cavity and maxillary sinuses, pterygopalatine fossa, inferior sphenoid sinus, posterior ethmoid sinus and anterior half of the clivus) and all high-risk neck nodes. CTV2 was defined as low-risk node regions below the CTV1. The respective planning target volumes (PTVs) were generated with a margin of $5 \mathrm{~mm}$ in all directions, but with a $2-3 \mathrm{~mm}$ margin when the corresponding CTV has overlapped with, or been close to, a critical structure, such as brain stem, spinal cord or optic nerves.

The prescribed radiation dose was delivered in 33 fractions, with 2.2Gy per fraction to PTVnx and PTVnd, 2.0Gy to PTV1 and 1.8Gy to PTV2. All patients were treated with one fraction daily for five days weekly. Radiation was delivered using a 6-MV linear accelerator (Varian Medical Systems, Palo Alto, CA, USA) once the treatment planning was approved. Furthermore, all patients were typically treated with cisplatin-based concurrent chemotherapy for sensibilization, infused intravenously at $75 \mathrm{mg} / \mathrm{m}^{2}, \mathrm{~d} 1-3$, two cycles totally.

\section{CBCT imaging and CBCT guidance protocol}

The kV-CBCT images were acquired using the Varian Medical Systems linear accelerator equipped with $\mathrm{kV}$ imaging capabilities (Varian Medical Systems, Palo Alto, CA, USA), with the following acquisition parameters: $\mathrm{kVp}, 100 \mathrm{kV}$; nominal milliamperes per frame, $10 \mathrm{~mA}$; nominal milliseconds, $10 \mathrm{~ms}$; $\mathrm{kV}$ collimator, s20; kV filter, f0; approximate frames, 361; and total angle, 200. Figure 1 shows the contrast between CBCT images and planning $\mathrm{CT}$ images of the nasopharynx in the transverse section. It is obvious that the CBCT images have the quality needed to perform an accurate image registration with planning $\mathrm{CT}$ images.

The $\mathrm{kV}$-CBCT scans were performed before the $1^{\text {st }}$, $6^{\text {th }}, 11^{\text {st }}, 16^{\text {th }}, 21^{\text {st }}$ and $26^{\text {th }}$ radiation time, respectively, after conventional positioning by calibrating the in-room lasers on the marked thermoplastic mask.

\section{Target delineation on CBCT images}

All fractionated CBCT images before the treatment were delivered to the Pinnacle 38.0 workstation, fused 
with the planning CT using the adaptive software. Target delineations (including the GTVnx, bilateral parotids and atlas) were contoured and confirmed by the same oncologist. Then the center of mass (COM) of the atlas and bilateral parotids, volumes of GTVnx and parotid glands, doses delivered to parotids were calculated automatically. The COM was a fictitious point in the physical system where quality was considered to set.

\section{Statistical analysis}

All target volumes delineated on fractionated CBCT images were recorded. Compared with the original GTVnx, it was considered as significant if the percentage of shrinking volume $(\Delta \mathrm{P}) \geq 50 \%(\Delta \mathrm{P}=\Delta \mathrm{GTV} /$ the volume of the original GTVnx, $\Delta \mathrm{GTV}=$ the volume of GTVnx on the corresponding fractionated CBCT images - the volume of the original GTVnx). Shrinking volumes and the percentage of bilateral parotids were calculated by the same means of the GTVnx. In addition, the space coordinates of all COM points were calculated automatically using the Pinnacle ${ }^{3} 8.0$ system. The atlas reference point was defined as the coordinate origin, with the space coordinate named point $\mathrm{P}_{1}\left(\mathrm{x}_{1}, \mathrm{y}_{1}, \mathrm{z}_{1}\right)$. And the distances between each parotid COM $\left[\mathrm{P}_{2}\left(\mathrm{x}_{2}, \mathrm{y}_{2}, \mathrm{z}_{2}\right)\right]$ and atlas COM were cacalculated using the formula $\left[\left(\mathrm{x}_{2}-\right.\right.$ $\left.\left.\mathrm{x}_{1}\right)^{2}+\left(\mathrm{y}_{2}-\mathrm{y}_{1}\right)^{2}+\left(\mathrm{z}_{2}-\mathrm{z}_{1}\right)^{2}\right]^{1 / 2}$. Then, the displacement was achieved comparing difference between two distances. $\mathrm{x} 2-\mathrm{x} 12+\mathrm{y} 2-\mathrm{y} 12+\mathrm{z} 2-\mathrm{z} 12$

The data were analyzed with SPSS version 17.0 statistical software (SPSS Inc., Chicago, IL, USA). The Pearson correlation coefficient was used to evaluate the correlation. Two-sided $P$-values of $<0.05$ were considered statistically significant.

\section{CONCLUSION}

In summary, during the IMRT of NPC, volumes of tumor targets and parotid glands varied significantly, with little displacement change in the center of parotid glands. This could cause a decrease radiation dose delivered to tumor targets and an increase radiation dose to parotid glands. So it is necessary to identify the time of repeat $\mathrm{CT}$ and replanning, and the time between the $21^{\text {st }}$ to $25^{\text {th }}$ radiations of IMRT is appropriate.

\section{Abbreviations}

NPC: nasopharyngeal carcinoma, RT: radiation therapy, IMRT: intensity-modulated radiation therapy, $\mathrm{kV}$ : kilovoltage, CBCT: cone beam computed tomography, GTV: gross tumor volume, GTVnx: GTV included the primary nasopharyngeal tumor, GTVnd: GTV included the involved lymph nodes, CTV: clinical target volume, PTV: planning target volume, $\mathrm{D}_{\text {mean }}$ : the planned mean dose, OARs: organs at risk, COM: the center of mass, $\triangle \mathrm{P}$ : the percentage of the shrinking volumes, $\Delta \mathrm{V}$ : the shrinking volumes of parotid glands, $\triangle \mathrm{GTV}$ : the shrinking volumes of GTVnx.

\section{Author contributions}

The authors contributions are the following: X-JG and Y-MW equally contributed to literature research, study design, patient recruitment, image delineation, data collection, data analysis, interpretation of findings and writing of the manuscript. H-MT and Z-J contributed to patient recruitment, image delineation and data collection. $\mathrm{B}-\mathrm{SL}$ (PhD, professor) contributed to interpretation of findings and critical edit of the manuscript. All authors read and approved the final manuscript.

\section{ACKNOWLEDGMENTS}

This work was supported by grants from the Projects of Medical And Health Technology Development Program In Shandong Province (No. 2015WS0158), the Natural Science Foundation of Shandong Province, People's Republic of China (No. ZR2014YL034).

\section{CONFLICTS OF INTEREST}

The authors have no conflict of interest.

\section{REFERENCES}

1. Al-Sarraf M, LeBlanc M, Giri PG, Fu KK, Cooper J, Vuong T, Forastiere AA, Adams G, Sakr WA, Schuller DE, Ensley JF. Chemoradiotherapy versus radiotherapy in patients with advanced nasopharyngeal.Cancer: Phase III randomized Intergroup study 0099. J Clin Oncol. 1998; 16:1310-1317.

2. Hunt MA, Zelefsky MJ, Wolden S, Chui CS, LoSasso T, Rosenzweig K, Chong L, Spirou SV, Fromme L, Lumley M, Amols HA, Ling CC, Leibel SA. Treatment planning and delivery of intensity-modulated radiation therapy for primary nasopharynx cancer. Int J Radiat Oncol Biol Phys. 2001; 49:623-632.

3. Kam M, Chau RM, Suen J, Choi PH, Teo PM. Intensitymodulated radiotherapy in nasopharyngeal carcinoma: Dosimetric advantage over conventional plans and feasibility of dose escalation. Int J Radiat Oncol Biol Phys. 2003; 56:145-157.

4. Lee N, Xia P, Quivey JM, Sultanem K, Poon I, Akazawa C, Akazawa P, Weinberg V, Fu KK. Intensity-modulated radiotherapy in the treatment of nasopharyngeal carcinoma: an update of the USCF experience. Int J Radiat Oncol Biol Phys. 2002; 53:12-22.

5. Lin S, Pan J, Han L, Zhang X, Liao X, Lu JJ. Nasopharyngeal carcinoma treated with reduced-volume 
intensity-modulated therapy: report on the 3-years outcome of a prospective series. Int J Radiat Oncol Biol Phys. 2009; 75:1071-1078.

6. Xing L, Lin Z, Donaldson SS, Le QT, Tate D, Goffinet DR, Wolden S, Ma L, Boyer AL . Dosimetric effects of patient displacement and collimator and gantry angle misalignment on intensity modulated radiation therapy. Radiother Oncol. 2000; 56:97-108.

7. Wang J, Bai S, Chen N, Xu F, Jiang X, Li Y, Xu Q, Shen Y, Zhang H, Gong Y, Zhong R, Jiang Q. The clinical feasibility and effect of online cone beam computer tomography-guided intensity-modulated radiotherapy for nasopharyngeal cancer. Radiother Oncol. 2009; 90:221227.

8. Barker JL Jr, Garden AS, Ang KK, O’Daniel JC, Wang H, Court LE, Morrison WH, Rosenthal DI, Chao KS, Tucker SL, Mohan R, Dong L. Quantification of volumetric and geometric changes occurring during fractionated radiotherapy for head-and-neck cancer using an integrated CT/linear accelerator system. Int J Radiat Oncol Biol Phys. 2004; 59:960-970.

9. Hansen EK, Bueci MK, Quivey JM, Weinberg V, Xia P. Repeat $\mathrm{C} \mathrm{T}$ imaging and replanning during the course of IMRT for head-and-neck cancer. Int J Radiat Oncol Biol Phys. 2006; 64:355-362.

10. Wang W, Yang H, Hu W, Shan G, Ding W, Yu C, Wang B, Wang X, Xu Q. Clinical study of the necessity of replanning before the 25th fraction during the course of intensitymodulated radiotherapy for patients with nasopharyngeal carcinoma. Int J Radiat Oncol Biol Phys. 2010; 77:617-621.

11. Han C, Chen YJ, Liu A, Schultheiss TE, Wong JY. Actual dose variation of parotid glands and spinal cord for nasopharyngeal cancer patients during radiotherapy. Int $\mathrm{J}$ Radiat Oncol Biol Phys. 2008; 70:1256-1262.

12. Yang BB, Wang J, Zhong RM, Jiang QF, Chen NY, Bai $\mathrm{S}$. [Volumetric and geometric changes of parotids occuring during IMRT for nasopharyngeal carcinoma (NPC) using daily CBCT]. [Article in Chinese]. Sichuan Da Xue Xue Bao Yi Xue Ban. 2010; 41:1024-1028.

13. Nutting CM, Morden JP, Harrington KJ, Urbano TG, Bhide SA, Clark C, Miles EA, Miah AB, Newbold K, Tanay M, Adab F, Jefferies SJ, Scrase C, et al. Parotidsparing intensity modulated conventional radiotherapy in head and neck cancer (PARSPORT): a phase 3 multicentre randomised controlled trial. Lancet Oncol. 2011; 11:127136.
14. Lee N, Harris J, Garden AS, Straube W, Glisson B, Xia P, Bosch W, Morrison WH, Quivey J, Thorstad W, Jones $\mathrm{C}$, Ang KK. Intensity-modulated radiation therapy with or without chemotherapy for nasopharyngeal carcinoma: radiation therapy oncology group phase II trial 0225 . J Clin Oncol. 2009; 27:3684-3690.

15. Ren G, Xu SP, Du L, Feng LC, Qu BL, Liu HX, Xie $\mathrm{CB}, \mathrm{Ma}$ L. Actual anatomical and dosimetric changes of parotid glands in nasopharyngeal carcinoma patients during intensity modulated radiation therapy. Biomed Res Int. 2015; 2015:670327.

16. Broggi S, Fiorino C, Dell'Oca I, Dinapoli N, Paiusco M, Muraglia A, Maggiulli E, Ricchetti F, Valentini V, Sanguineti G, Cattaneo GM, Di Muzio N, Calandrino R. A two-variable linear model of parotid shrinkage during IMRT for head and neck cancer. Radiother Oncol. 2010; 94:206-212.

17. Xia P, Fu KK, Wong GF, Akazawa C, Verhey LJ. Comparison of treatment plans involving intensitymodulated radiotherapy for nasopharyngeal carcinoma. Int J Radiat Oncol Biol Phys. 2000; 48:329-337.

18. Yao WR, Xu SP, Liu B, Cao XT, Ren G, Du L, Zhou FG, Feng LC, Qu BL, Xie CB, Ma L. Replanning criteria and timing definition for parotid protection-based adaptive radiation therapy in nasopharyngeal carcinoma. Biomed Res Int. 2015; 2015:476383.

19. Robar JL, Day A, Clancey J, Kelly R, Yewondwossen M, Hollenhorst H, Rajaraman M, Wilke D. Spatial and dosimetric variability of organs at risk in head-and-neck intensitymodulated radiotherapy. Int J Radiat Oncol Biol Phys. 2007; 68:1121-1130.

20. Vasquez Osorio EM, Hoogeman MS, Al-Mamgani A, Teguh DN, Levendag PC, Heijmen BJ. Local anatomic changes in parotid and submandibular glands during radiotherapy for oropharynx cancer and correlation with dose, studied in detail with nonrigid registratio. Int J Radiat Oncol Biol Phys. 2008; 70:875-882. 